\title{
Efficient Parallel Discrete Event Simulation on Cloud/Virtual Machine Platforms
}

\author{
SRIKANTH B. YOGINATH and KALYAN S. PERUMALLA, Oak Ridge National Laboratory
}

Cloud and Virtual Machine (VM) technologies present new challenges with respect to performance and monetary cost in executing parallel discrete event simulation (PDES) applications. Due to the introduction of overall cost as a metric, the traditional use of the highest-end computing configuration is no longer the most obvious choice. Moreover, the unique runtime dynamics and configuration choices of Cloud and VM platforms introduce new design considerations and runtime characteristics specific to PDES over Cloud/VMs. Here, an empirical study is presented to help understand the dynamics, trends, and trade-offs in executing PDES on Cloud/VM platforms. Performance and cost measures obtained from multiple PDES applications executed on the Amazon EC2 Cloud and on a high-end VM host machine reveal new, counterintuitive VM-PDES dynamics and guidelines. One of the critical aspects uncovered is the fundamental mismatch in hypervisor scheduler policies designed for general Cloud workloads versus the virtual time ordering needed for PDES workloads. This insight is supported by experimental data revealing the gross deterioration in PDES performance traceable to VM scheduling policy. To overcome this fundamental problem, the design and implementation of a new deadlock-free scheduler algorithm are presented, optimized specifically for PDES applications on VMs. The scalability of our scheduler has been tested in up to 128 VMs multiplexed on 32 cores, showing significant improvement in the runtime relative to the default Cloud/VM scheduler. The observations, algorithmic design, and results are timely for emerging Cloud/VM-based installations, highlighting the need for PDES-specific support in high-performance discrete event simulations on Cloud/VM platforms.

Categories and Subject Descriptors: C.2.2 [Computer Systems Organization]: Computer-Communication Networks-Network protocols; C.5.1 [Computer Systems Organization]: Computer System Implementation-Large and medium computers; I.6.1 [Computing Methodologies]: Simulation and Modeling-Discrete; I.6.8 [Computing Methodologies]: Simulation and Modeling-Types of simulation (discrete event, parallel)

General Terms: Algorithms, Design, Experimentation, Performance

Additional Key Words and Phrases: Parallel discrete event simulation, time warp, global virtual time, virtual machines, scheduler

ACM Reference Format:

Srikanth B. Yoginath and Kalyan S. Perumalla. 2015. Efficient parallel discrete event simulation on Cloud/Virtual machine platforms. ACM Trans. Model. Comput. Simul. 26, 1, Article 5 (June 2015), 26 pages.

DOI: http://dx.doi.org/10.1145/2746232

\section{INTRODUCTION}

\subsection{Cloud/VMs as PDES Execution Platform}

Parallel computing platforms based on virtualization technologies, such as commercial Cloud offerings, have emerged lately, and are seen as a good alternative to native

Authors' address: S. B. Yoginath and K. S. Perumalla, 1 Bethel Valley Road, Oak Ridge, TN 37831.

Permission to make digital or hard copies of part or all of this work for personal or classroom use is granted without fee provided that copies are not made or distributed for profit or commercial advantage and that copies show this notice on the first page or initial screen of a display along with the full citation. Copyrights for components of this work owned by others than ACM must be honored. Abstracting with credit is permitted. To copy otherwise, to republish, to post on servers, to redistribute to lists, or to use any component of this work in other works requires prior specific permission and/or a fee. Permissions may be requested from Publications Dept., ACM, Inc., 2 Penn Plaza, Suite 701, New York, NY 10121-0701 USA, fax +1 (212) 869-0481, or permissions@acm.org.

(c) 2015 ACM 1049-3301/2015/06-ART5 $\$ 15.00$

DOI: http://dx.doi.org/10.1145/2746232 
execution directly on specific parallel computing hardware. There are several benefits to using the virtualization layer, making such platforms very appealing as an alternative approach to execute parallel computing tasks. In the context of parallel discrete event simulation (PDES), the benefits include the following:

- The ability of the virtualization system to simultaneously host and execute multiple distinct operating systems (OS) enables PDES applications to utilize a mixture of simulation components written for disparate OS platforms.

- The ability to oversubscribe physical resources (i.e., multiplex larger number of Virtual Machines [VMs] than available physical computing resources) allows the PDES applications to dynamically grow and shrink the number of physical resources as the resources become available or unavailable, respectively.

-The dynamic imbalances in event loads inherent in most PDES applications can be efficiently addressed using the process migration feature of the virtual systems.

- The fault tolerance features supported at the level of VMs in concert with the VM migration feature also automatically helps in achieving fault tolerance for PDES applications.

\subsection{Issues and Challenges}

PDES has traditionally assumed execution at the highest end of the computing platform available to the user. However, the choice is not so straightforward in Cloud computing due to the nonlinear relation between actual parallel runtime and the total cost (charged to the user) for the host hardware.

For example, suppose that a multicore computing node has 32 cores on which a PDES with 32 concurrent simulation loops is to be executed. Generally speaking, traditional PDES maps one simulation loop to one native processor. However, with Cloud computing, the monetary charge for such a direct mapping (i.e., a virtual machine with 32 virtual cores) is typically much larger than the total monetary charge for aggregates of smaller units (i.e., 32 virtual machines each with only 1 virtual core).

Nonlinear Cost Structure. The nonlinear cost structure is fundamentally rooted in the principles of economies of scale - the Cloud hosting company gains flexibility of movement and multiplexed mapping of smaller logical units over larger hosting units, ultimately translating to monetary margins. Moreover, a high-end multicore configuration on native hardware is not the same as a high-end multicore configuration on virtual hardware because the interprocessor (inter-VM) network appears in software for VMs, but in "silicon and copper" for native hardware. The aggregate interprocessor bandwidth is significantly different between the virtualized (software) network and insilico (hardware) network (performance analysis supporting this insight is presented later).

Multiplexing Ratio. Given that multiple VMs must be used to avoid the high price of a single many-core VM, the performance of PDES execution now becomes dependent on the scheduling order of the VMs (virtual cores) on the host (real hardware cores). This puts PDES performance at the mercy of the hypervisor scheduler's decisions. When the multiplexing ratio (ratio of sum of virtual cores across all VMs to the sum of actual physical cores) even fractionally exceeds unity, the PDES execution becomes vastly suboptimal. In all Cloud offerings, this multiplexing ratio can (and will very often) exceed unity dynamically at runtime. Thus, we have a conflict: one-to-one mapping (multiplexing ratio of unity or smaller) incurs a higher monetary cost, but increasing the multiplexing ratio causes a scheduling problem, and increases the runtime, thereby stealing any monetary gains.

Scheduling Problem. The conflict arises due to the hypervisor scheduler: the default schedulers designed for general Cloud workloads are a mismatch to PDES workloads. 
The hypervisor is a critical component of the virtualized system, enabling the execution of multiple VMs on the same physical machine. To support the largest class of applications on the Cloud, a fair-sharing scheme is employed by the hypervisor for sharing the physical processors among the VMs. The concept of fair sharing works best either when the VMs execute relatively independently of each other or when the concurrency across VMs is fully realized via uniform sharing of computational cycles. This property holds in the vast majority of applications in general. However, in PDES, fair-share scheduling does not match the required scheduling order and, in fact, may run counter to the required order of scheduling. This mismatch arises from the fundamental aspect of interprocessor dependency in PDES, namely, the basis on the global simulation time line.

Virtual Time-Based Scheduling. In PDES, the simulation time advances with the processing of timestamped simulation events. In general, the number of events processed in a PDES application varies dynamically during the simulation execution (i.e., across simulation time), and also varies across processors. This implies that the amount of computation cycles consumed by a processor for event computation does not have any specific, direct correlation with its simulation time. A processor that has few events to process within a simulation time window ends up consuming few computational cycles. It is not ready to process events belonging to the simulation-time future until other processors have executed their events and advanced their local simulation time. However, a fair-share scheduler would bias the scheduling towards this lightly loaded processor (since it has consumed fewer cycles) and penalize the processors that do, in fact, need more cycles to process their remaining events within that time window. This type of operation works against the actual time-based dependencies across processors, and can dramatically deteriorate the overall performance of the PDES application. This type of deterioration occurs when conservative synchronization is used. Similar arguments hold for optimistic synchronization, but, in this case, the deterioration can also arise in the form of an increase in the number of rollbacks. The only way to solve this problem is to design a new scheduler that is aware of, and accounts for, the simulation time of each VM, and schedule each in a least-simulation-time-first order.

A final consideration is that a scheduling algorithm based solely on least-simulationtime-first-order is susceptible to deadlocks that need to be resolved and implemented in a scalable manner with respect to the number of VMs multiplexed by the hypervisor. Thus, a new, deadlock-free, scalable hypervisor scheduling algorithm is needed to deliver the most efficient execution of PDES on Cloud/VM platforms.

\subsection{Contributions}

This article is an extended version of our previous work [Yoginath and Perumalla 2013a, $2013 \mathrm{~b}$. This article adds (a) a new hypervisor scheduler algorithm for PDES execution on VM/Cloud platforms, (b) design and implementation specifics of the new scheduler in Xen hypervisor, (c) results from the performance evaluation of the new PDES scheduler implementation using an expanded set of benchmark applications with wide-ranging scenario configurations, (d) performance results from scaling benchmark applications to over $128 \mathrm{VMs}$, and (e) performance comparison of the new VM scheduler with native Linux.

\subsection{Organization}

The rest of the article is organized as follows. In Section 2, a brief background is provided on the major concepts used in the work. In Section 3, the design of the PDES-specific VM scheduler algorithm is presented, followed by the details of its implementation in Section 4. A detailed performance evaluation is presented in Section 5. 
Related work is covered in Section 6 . The results are summarized in Section 7, followed by conclusions and future work in Section 8 .

\section{BACKGROUND}

In this section, a brief overview is provided on VMs, Cloud computing, the Xen hypervisor, hypervisor scheduling, and PDES execution.

\subsection{Virtual Machines}

The concept of virtualization has been realized at different levels of the computer systems architecture. At the hardware level, two methodologies are used for virtualization: full virtualization and para-virtualization. Although they are similar in functionality, they differ in the means to realizing virtualization. Both the methodologies run on the top of the hardware by pushing the OS above them and make use of highly configurable VMs comprising virtual peripheral I/O components. Para-virtualization differs from full virtualization in that it requires the modification of the guest OS kernel, while the full virtualization can host any OS without modifications.

The VMware ESX Server, the Xen Hypervisor, and the Microsoft Hyper-V hypervisor are examples of popular VM systems. The VMware ESX Server hypervisor was principally designed to support full virtualization. The Xen [Chisnall 2007; Matthews et al. 2008] hypervisor started with the concept of para-virtualization, but currently also supports full virtualization. The Microsoft Hyper-V hypervisor also supports full virtualization. The concepts developed in this article apply equally well to all these VM systems because all share the fundamental concept of multiplexing many virtual resources on fewer physical resources; it is the multiplexing of virtual processor cores over real processor cores that creates a fundamental runtime problem of correctness and runtime efficiency of PDES over VMs.

Currently, applications based on virtualization technology span from single-user desktops to huge data centers. On the lower end, virtualization allows desktop users to concurrently host multiple OS instances on the same hardware. On a larger scale, VMs can be moved from one hypervisor (or device) to another even while the VMs are actively running. This capability for mobility is used to support many advantageous features of Cloud computing, including load balancing, fault tolerance, and economical hosting of computing/storage services.

\subsection{Cloud Computing}

A very attractive product for both business operators and users alike arose from tapping virtualization technology through Internet services, which famously came to be known as Cloud computing. Infrastructure as a Service (IAAS), Platform as a Service (PAAS), Software as a Service (SAAS) and Network as a Service (NAAS) are prominent among the types of services offered currently by the Cloud computing service vendors [Mell and Grance 2011]. Apart from IAAS, the other services are completely unaware of the physical hardware on which they are executing. Exploiting the unlikeliness of 100\% resource utilization from all clients at all times, Cloud operators multiplex VMs on limited resources and hence are able to provide easy accessibility to large computing resources at an impressively competitive price.

\subsection{Xen Hypervisor}

The Xen hypervisor is a popular open-source industry standard for virtualization, supporting several architectures including x86, x86-64, IA64, and ARM, and guest OS types including Windows, Linux, Solaris, and various versions of BSD OS. Figure 1 shows a schematic of guests running on the Xen hypervisor. Xen refers to VMs as Guest Domains or DOMs. Each DOM is identified by its DOM-ID. The first DOM, 


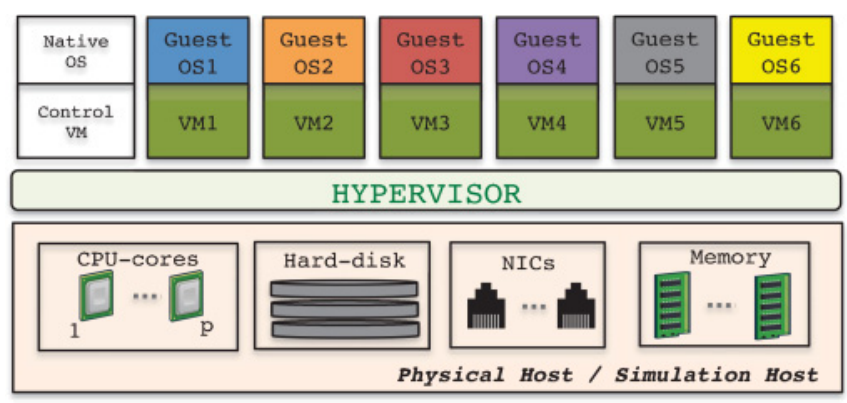

Fig. 1. Xen hypervisor.

DOM-0, possesses special hardware privileges. Such privileges are not provided to other user DOMs, which are generically referred to as DOMUs. Each DOM has its own set of virtual devices, including virtual multiprocessors called virtual CPUs (VCPUs). System administration tasks such as suspension, resumption, and migration of DOMs are managed via DOM0.

\subsection{Hypervisor Scheduling}

Among the shared resources multiplexed by the hypervisor, the physical processor cycles are especially important for PDES. To seamlessly share the physical CPU (PCPU) resources among the virtual CPUs (VCPUs), the hypervisor contains a scheduler that allots PCPU cycles to the VCPUs using a scheduling policy. The hypervisor's scheduling is distinct from multithreading in operating systems hosted in the VMs over the hypervisor [Chisnall 2007]. Essentially, there exist three scheduling tiers in Xen: (a) a userspace threading library schedules user-space threads over OS-level (kernel) threads within a VM, (b) every guest OS schedules its kernel threads to VCPUs, and (c) the hypervisor schedules the VCPUs over the PCPUs. The focus of this article is on the lowest layer, namely, the hypervisor-level mapping of VCPUs to PCPUs.

\subsection{Credit Scheduler of Xen}

The credit-based scheduler of Xen (CSX) is Xen's default hypervisor scheduler based on the principle of fair sharing. CSX uses a concept of credits for every DOM; these credits are expended as the DOM's VCPUs are scheduled for execution. It provides control to the user to alter the configuration of scheduling through parameters called weight and cap. While the weight value determines the share of PCPU cycles a DOM's VCPU gets with respect to the VCPUs of other DOMs, the cap value restricts the utilizable PCPU cycles by a DOM's VCPU. By default, the weight value for all DOMs is 256 and cap is 0 , providing a fair CPU allocation to all of the DOMs. This scheduler is very widely used, and works excellently for a large variety of virtualization uses. However, under overloaded conditions (number of VCPUs > number of PCPUs) and when the loads on DOMs are nonuniform (as is the case in PDES), this fair-share scheduling algorithm detrimentally affects the overall performance of the parallel application.

\subsection{PDES Model Execution}

In PDES, the model is divided into distinct independent virtual time lines referred to as logical processes (LPs). Each LP typically encapsulates a set of state variables of modeled entity. The time lines of LPs within and across processors are kept synchronized by the PDES simulation engine. PDES engines may support optimistic synchronization or conservative synchronization, or a combination. The runtime environment allows multiple LPs to be hosted per simulation loop (SL), and each SL is mapped to a single 


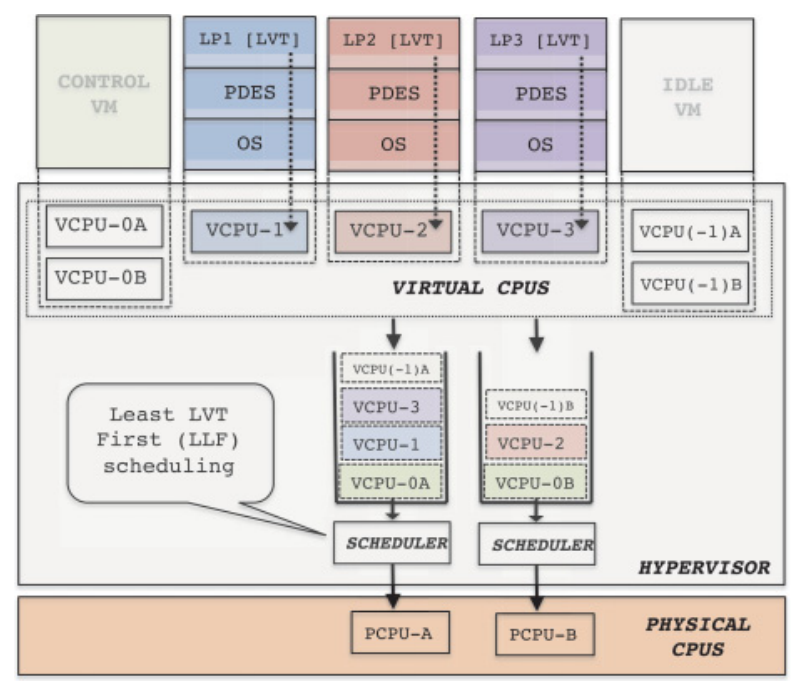

Fig. 2. PDES scheduler design.

processor core. When executed on VM, this implies that each SL is mapped to a single VCPU. Thus, the scheduling problem for PDES on VMs becomes one of (indirectly) scheduling SLs over PCPUs.

\section{PDES SCHEDULER DESIGN}

A hypervisor scheduler time-multiplexes many VCPUs and on to few PCPUs. To accomplish this, each PCPU maintains a run-queue, which is a list of VCPUs that share the PCPU resources, as shown in Figure 2. The hypervisor scheduler follows a systemdefined scheduling policy for dynamically mapping the VCPUs onto PCPUs. A fairshare policy is usually adopted by the default hypervisor schedulers to suit a wide range of workloads.

In PDES, the LP with the least value of local virtual time (LVT) affects the progress of the entire simulation. Hence, by prioritizing the VCPU that hosts the SL with the least LVT value, the runtime performance can be optimized. To achieve this, the SLs on different VMs need to communicate the LVT values to their corresponding VCPUs, so that the hypervisor scheduler can use that information to schedule the VCPUs onto the PCPUs.

Figure 2 shows the system architecture of a hypervisor-based execution platform customized for PDES applications. In the figure, for clarity, only a single LP is shown per SL and a single SL is shown per VCPU. However, any number of LPs per SL and any number of SLs per VCPU can be supported by our approach with simple modifications. As illustrated in Figure 2, the LVT of an LP is passed to the VCPU in its VM. The VCPU records the LVT inside the hypervisor's scheduler data structures as the VCPU LVT. The hypervisor scheduler uses the LVT values of the VCPUs in a Least-LVT-First (LLF) strategy during scheduling. With the LLF scheduling policy, the scheduler gives the highest priority to the VCPU with least the LVT value.

\subsection{Deadlocks from Purely LVT-Based VM Scheduling}

The VCPU scheduling based on a purely LLF-based policy leads to deadlock of PDES applications when the number of hosted VCPUs is greater than the available PCPUs. This is because the VCPUs with smaller VCPU LVT values (i.e., having a higher scheduling priority) would prevent the VCPUs with a higher VCPU LVT value from 
being scheduled for execution. Consequently, some of the SLs (those executing in VMs with higher LVT values) would never get a chance to participate in inter-SL time synchronization operations such as Global Virtual Time (GVT) or Lower Bound on incoming Time Stamp (LBTS) computation. Since GVT or LBTS constrain the progress of the simulation, the execution deadlocks. Note that this deadlock can arise for both conservative or optimistic synchronization. In conservative operation, simulation progress is critically dependent on global time guarantees, and hence no processor will be able to go past the most recently computed LBTS. Optimistic operation, although resilient to an extent to slow GVT progress rates, can also deadlock if/when the processor with the least LVT runs out of memory because fossil collection gets stalled when GVT computation does not progress.

\subsection{Deadlock Condition}

In this section, we reason the presence of deadlocks that arise in a purely LLFbased scheduler. For a system to deadlock, four conditions are necessary and sufficient [Coffman et al. 1971]:

-Mutual exclusion: Tasks claim exclusive control of the resources.

- Hold and wait condition: Tasks hold resources already allocated to them while waiting for additional resources.

-No preemption: Resources cannot be forcibly removed from the tasks holding them until the resources are used to completion.

- Circular wait: A circular chain of tasks exists, such that each task holds one or more resources that are being requested by the next task in the chain.

All these conditions arise with PDES over pure the LLF hypervisor scheduler as follows.

Mutual Exclusion. At runtime, only one VCPU can execute at any given time on a PCPU. When a VCPU is scheduled, no other VCPU can get computational cycles. The scheduled VCPU always has exclusive control of the PCPU resource. This satisfies the mutual exclusion criterion for deadlock.

Hold and Wait. As long as the scheduled VCPU has the least LVT, it is always assured of coming back to the PCPU even after its timeslice is exhausted. If the LVT of such a VCPU (with least LVT) does not progress, that VCPU holds up the PCPU resource (HOLD). The PCPU resource is indefinitely held up by this least-LVT VCPU (WAIT) because it is waiting for another processor to participate in GVT/LBTS computation. This will not be resolved because at least one of the SLs (with a larger LVT) does not participate in the GVT computation due to nonavailability of PCPU compute cycles.

No-Preemption Condition. Since the hypervisor scheduler strictly follows least-LVT first order of scheduling, it never preempts the least-LVT VCPU with other VCPUs.

Circular Wait Condition. The LVT values to each of the VCPUs are passed from the PDES application. To ensure the progress of the simulation, each of the SLs need to regularly exchange their LVTs with their peer SLs to compute the GVT. For the GVT computation, SLs need PCPU cycles. When the number of VCPUs is greater than number of PCPUs, some VCPUs do get PCPU cycles. Hence, the least-LVT VCPU waits for the GVT message from its peer, while its peer VCPUs (with higher LVT value) wait on the least-LVT VCPU to release the PCPU resource (to participate in GVT computation). Thus, a circular wait condition emerges.

\subsection{Deadlock Resolution}

The deadlock does not occur when at least one of the aforementioned conditions is violated. Hence, a simple way to break the deadlock is through preemption. In the 
hypervisor scheduling process, this can be done by occasionally ignoring the application-supplied LVT and increasing the LVT value of the VCPU to a large value. One simple algorithm to achieve this is to make the VCPU LVTs toggle between the actual LVT and a very large LVT value in regular scheduling intervals. By such toggling, the VCPUs self-preempt themselves momentarily. This simple algorithm breaks the deadlock and ensures that all the SLs get the necessary PCPU cycles to participate in GVT or LBTS algorithms, as demonstrated in Yoginath and Perumalla [2013b]. However, this method is inefficient because of the significantly large number of selfpreemptions. This simple algorithm can be optimized by relaxing it into a counter-based approach, wherein every VCPU maintains a counter in addition to its VCPU LVT. This counter in the VCPU is incremented whenever it is bypassed during scheduling by another VCPU with a lower LVT. When the counter of any VCPU reaches a prespecified threshold (empirically set), this VCPU preempts others in the run queue to break the deadlock.

\subsection{Counter-Based Algorithm to Resolve Deadlock}

Algorithm 1 gives the pseudo-code for our new deadlock-free PDES-specific hypervisor scheduler algorithm. As mentioned previously, the hypervisor scheduler inserts the currently executed VCPU $v_{c}$ into the run queue and picks a new VCPU $v_{n}$ for scheduling onto the PCPU. The hypervisor scheduler servicing the interrupt of the PCPU performs this scheduling action continuously. Algorithm 1 starts executing on PCPU $p$ with $v_{c}$ as input and determines $v_{n}$ as the output. The process of selecting the $v_{n}$ follows the LLF principle for PSX, modified for deadlock avoidance and for minimizing interprocessor synchronization (locking).

The algorithm starts by updating the VCPU LVT $T\left[v_{c}\right]$ value of the $v_{c}$ before inserting it into the interrupted PCPU $p$ 's run queue $(R Q[p])$ following the LLF principle (Lines 2 to 4$)$. A GVT counter $(g[1: V])$ is maintained for each VCPU. During insertion of $v_{c}$ into the local run queue, the GVT counters of all VCPUs being preceded in the local queue $R Q[p]$ are incremented (i.e., those VCPUs whose LVT is greater than $v_{c}$ 's [Lines 5 to 7]).

Next, the VCPU to be scheduled next $\left(v_{n}\right)$ is determined as follows. The run queue $R Q[p]$ is first searched to find if any VCPU is starving for cycles. This is indicated by a GVT counter value exceeding the GVT threshold $G$. If any VCPU satisfying this preemption condition is found, that VCPU is selected as $v_{n}$ (Lines 9 to 14), and scheduled next after resetting its GVT counter $g[p]$ to zero (Line 37). If no such VCPU is found, then an effort is made to find a VCPU with a lower LVT across all the PCPU run queues (Lines 15 to 36). In this code segment, first the least LVT VCPU of $R Q[p]$ is picked as the default candidate $v_{n}$ and its LVT is compared with that of the VCPUs at the head of run queues of other PCPUs. If this search is unsuccessful, the local candidate is returned as $v_{n}$. Otherwise, the VCPU $v_{r}$ stolen from the peer PCPU run queue $\left(R Q\left[p^{\prime}\right]\right)$ is returned as $v_{n}$. Note that code segment in between (Lines 32 to 35) increments the GVT counter of all the VCPUs in $R Q[p]$ on successfully stealing the VCPU $v_{r}$. Not incrementing the GVT counter after stealing of lower LVT VCPU $v_{r}$ results in scenarios wherein the lower LVT VCPUs may be continuously exchanged between the PCPU run queues without altering the GVT counters of the VCPUs. Hence, not incrementing a GVT counter of all VCPUs in $R Q[p]$ after successfully stealing makes the deadlock persist. Finally, the GVT counter of the $v_{n}$ is reset before it is scheduled on the PCPU.

\section{PDES SCHEDULER IMPLEMENTATION}

To realize the PDES optimized hypervisor scheduler, we require (a) each $\mu$ sik kernel instance to independently communicate its LVT value to the Xen scheduler, and (b) a new Xen hypervisor scheduler that utilizes the LVTs to optimize the compute resource 









Fig. 3. PSX shared_info data structure.

sharing specifically for PDES applications. Also, note that the PDES specific hypervisor scheduler should be active only during the execution of the PDES application. To realize this, we need at least two different modes of hypervisor scheduler operations. We refer to the mode of operation during PDES execution as simulation mode, which otherwise is referred to be in a normal mode of operation.

\subsection{Communicate LVT to Hypervisor Scheduler}

To efficiently communicate LVT to the hypervisor, support from the guest-OS kernel is necessary. The guest-OS kernel should also impart the obtained application-level information to the hypervisor. Further, the PDES $\mu$ sik library should also be modified to communicate the change in hypervisor scheduler mode of operation, and the LVT value, to the guest-OS kernel.

4.1.1. Linux KernelModifications. Each guest OS maintains a shared_info page (Figure 3), which is dynamically updated as the system runs. We added two fields, simtime and switch_scheduler, to the shared_info data structure. The simtime field is used to record the LVT from the SL and the switch_scheduler flag indicates the switch between different modes of scheduler operation. To send the LVT information from the application level, we implemented a system call for our guest OS (Linux). This system call allows the LVT information to transit from user space to kernel space, and here the LVT value is written into the simtime of shared_info data structure of the host DOM. This shared_info data structure is accessed by the hypervisor during scheduling.

4.1.2. Modifications to the PDES Engine $\mu$ sik. Every $\mu$ sik SL maintains a variety of simulation times based on its event-processing state at any given moment. They are distinctly classified into four classes: committed, committable, processable, and emittable [Perumalla 2005]. We can transmit any of these LVT values to the hypervisor. In practice, we observed that the use of the earliest_committable_time_stamp resulted in better performance than the others, and hence, this simulation time value was used in all our experiments.

During simulation initialization, the $\mu$ sik library sets switch_scheduler in shared_info of its host DOM to true, using our system call. The scheduler reads this variable to change its mode of operation from normal mode to simulation mode. Similarly, during the termination of simulation, the switch_scheduler is set to false, suggesting to the scheduler to revert back to its normal mode of operation. The same system call is used by the $\mu$ sik library to write its SL's LVT value to simtime of the shared_info of host DOM. 


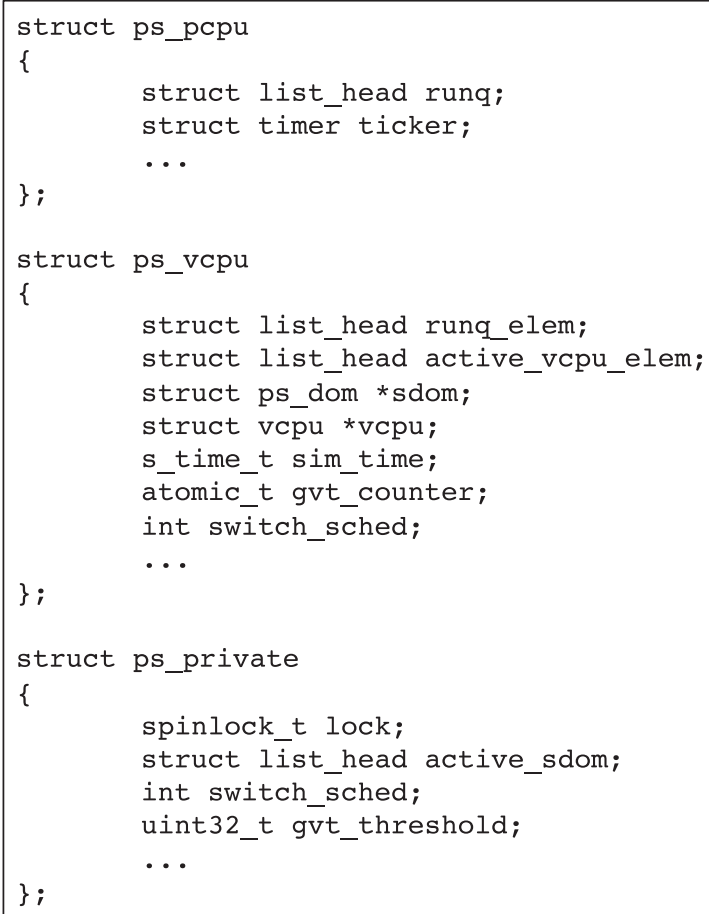

Fig. 4. PSX VCPU, PCPU, and global ps_private data structures.

\subsection{LVT-Based PDES Hypervisor Scheduler}

The PDES Scheduler for Xen (PSX) scheduler replaces the default CSX in scheduling the VCPU onto the PCPU. The strategy that we use to replace the scheduler is similar to the one presented by Yoginath and Perumalla [2011]. ps_private (Figure 4) is the structure of the global data-structure object that the PSX scheduler maintains. The switch_sched variable of the ps_private object is updated during VCPU scheduling after the corresponding value is read from sharedinfo by the VCPU from its relevant DOM. Setting the switch_sched in the PSX's ps_private global variable enables PSX to switch from normal mode to simulation mode and vice versa.

4.2.1. Scheduling in Normal Mode. The scheduler is said to be in normal mode if the switch_sched (of ps_private data structure; Figure 4) is false. This corresponds to the mode in which the VMs are booted and operational, but no PDES run is active (hence LVT-based scheduling is undefined). In this mode of operation, PSX maintains the sim_time (VCPU data structure; Figure 4) of all DOM0 VCPUs lower than all the DOMUs. In the normal mode, all guest-DOM VCPUs have their sim_time initialized to a constant 1 , while DOM0 VCPUs have their sim_times initialized to a constant 0 . Only after the switch_sched is set to true by PDES SL, the sim_time value of the relevant VCPU is updated after reading the shared_info. However, the sim_time of VCPUs of DOM0 continues to be 0 even after switching to simulation mode.

4.2.2. Scheduling in Simulation Mode. The hypervisor switches to the simulation mode after the PDES execution is started on all VMs. Each PCPU maintains an $R Q$ (priority queue) as shown in Figure 4 and, in the simulation mode, PSX en-queues the VCPUs to be scheduled in LLF priority. We use the LVT as the VCPU priority; the lower the sim_time (VCPU data structure; Figure 4), the higher is its priority in the $R Q$, 
Table I. EC2 VM Specifications

\begin{tabular}{|l|l|}
\hline m1.small & 1-core VM with compute power of 1 ECU with memory of 1.7GB \\
m1.medium & 1-core VM with compute power of 2 ECUs with $3.7 \mathrm{~GB}$ memory \\
m1.large & 2-core VM with compute power of 4 ECUs with memory of 7.5GB \\
$m 1 . x$ large & 4-core VM with compute power of 8 ECUs with memory of 15GB \\
$m 3.2 x$ large & 8-core VM with compute power of 26 ECUs with memory of $30 \mathrm{~GB}$ \\
hs.8xlarge & 16-core VM with compute power of 35 ECUs with memory of 117GB \\
\hline
\end{tabular}

hence the earlier it is picked by PSX to allocate compute resource. The scheduler allots a tick amount of PCPU cycles for the selected VCPU. Also, note that the sim_time corresponding to the VCPUs of the DOM0 is always maintained to be lower than that of other VCPUs regardless of the PSX's mode of operation. This guarantees that DOM0 VCPUs are always preferred over the other VCPUs, which in turn ensures a good and responsive user interactivity with DOM0 before, during, and after PDES executions.

\section{PERFORMANCE EVALUATION}

\subsection{Hardware}

5.1.1. Local Test Platform (LTP). LTP is our custom-built machine with a Supermicro H8DG6-F motherboard supporting two 16-core (32 cores in total) AMD Opteron 6276 processors at $2.3 \mathrm{GHz}$, sharing $256 \mathrm{~GB}$ of memory, Intel Solid State Drive 240GB, and a 6TB Seagate constellation comprising 2 SAS drives configured as RAID-0. Ubuntu 12.10 runs with Linux 3.7.1 kernel runs as DOM0 and DOMUs, over Xen 4.2.0 hypervisor. All DOMUs are para-virtual and networked using a software bridge in DOM-0. DOM-0 is configured to use 10GB of memory, and the guest DOMs were configured to use at least $1 \mathrm{~GB}$ memories each, which were increased as necessitated by the application benchmarks. Each guest DOM uses 2GB of LVM-based hard disk created over SAS drives, while the DOM-0 uses an entire Solid State Drive (SSD). OpenMPI 1.6.3 (built using gec-4.7.2) was used to build the simulation engine and its applications. A machine file listing the IP addresses of the VMs was used along with mpirun utility of OpenMPI to launch the MPI-based PDES applications onto VMs. The 1ms tick size was used with both CSX and PSX schedulers.

5.1.2. Amazon EC2. We also ran our benchmarks on Amazon's EC2 Cloud platform. We built a cluster of para-virtual VM instances of Ubuntu 12.04 LTS. Table I lists the VMs using the clusters that were built to run the performance benchmarks. In Table I, the term ECU refers to an EC2 Compute Unit, which is an abstraction defined and supported by Amazon as a normalization mechanism to provide a variety of virtual computation units independent of the actual physical hardware support that they use/maintain/upgrade without user intervention. OpenMPI 1.6.3 was built on the virtual instance, which was used to build the simulation engine and all the PDES applications. A machine file listing the DNS names of the allotted instances was used to launch the MPI-based PDES applications using mpirun.

\subsection{Software}

Three $\mu$ sik library-based application benchmarks-PHOLD [Fujimoto 1990] (a synthetic PDES application generally used for performance evaluation), PDES-based Disease Spread simulation [Perumalla and Seal 2012], and SCATTER [Yoginath and Perumalla 2008, 2009] (a PDES-based vehicular traffic simulation application)—were used for our performance studies. Various abbreviations used by the PDES applications and performance graphs have been consolidated in Table II. 
Table II. Abbreviations Used by PDES Applications

\begin{tabular}{|l|l|}
\hline$C S X$ & Credit Scheduler of Xen \\
$P S X$ & PDES Scheduler for Xen \\
$C O N S$ & PDES using conservative synchronization scheme \\
$O P T$ & PDES using optimistic synchronization scheme \\
$N L P$ & Number of LPs per simulation loop (SL) \\
$N M S G$ & Number of messages generated per LP \\
$L O C$ & Percent of traffic generated for local LPs (within same SL) \\
$L A$ & Lookahead \\
\hline
\end{tabular}

5.2.1. PHOLD Benchmark. This is a widely used synthetic benchmark for performance evaluation in the PDES community. This PDES application randomly exchanges a specified set of messages between the LPs. The $\mu$ sik implementation of PHOLD allows the exercising of a wide variety of options in its execution. The NLP, NMSG, LA, LOC, and synchronization techniques comprising CONS and OPT were varied to realize a range of simulation scenarios.

5.2.2. Disease Spread Benchmark (DSB). This is an epidemiological disease spread PDES application [Perumalla and Seal 2012] that uses a discrete event approach to model the propagation of a disease in a population of individuals across locations and across regions (aggregates of locations). Each region is mapped to an SL and each location is housed in an LP. Multiple individuals are instantiated at each location; they not only interact with individuals within the same location but also periodically (conforming to an individual-specific time distribution function) move from one location to another within and across regions. The scenario configuration parameters for this application are the same as for PHOLD, for which NLP refers to number of locations in a region (SL), NMSG refers to population/location, and LOC refers to percentage of population movements within the same region.

5.2.3. SCATTER Benchmark. This application is a discrete-event formulation and a parallel execution framework for vehicular traffic simulation. A simulation scenario is set up by reading an input file that specifies the road-network structure, number of lanes, speed limit, source nodes, sink nodes, vehicle generation rate, traffic light timings, and other relevant information. Dijkstra's shortest-path algorithm is used to direct a vehicle to its destination.

\subsection{CSX Performance Characteristics}

Before presenting the performance comparison of the PSX over the default CSX, we touch upon certain interesting aspects of CSX performance. Some information presented here is borrowed from our prior detailed study [Yoginath and Perumalla 2013a].

5.3.1. Native vs. VM. In Figure 5, we compare the PDES performance of PHOLD and DSB on a native Linux and VMs over the same hardware platform. Performance on VM, which can be either privileged (DOM0) or nonprivileged (DOMU), is also presented. The PHOLD scenarios were configured to use $100 \mathrm{LPs} / \mathrm{SL}, 100$ and 1000 messages/LP, with 32 SLs for $50 \%$ and $90 \%$ LOC values. The DSB scenario involved simulation of disease spread across 320 locations among a population of 320,000 for 7 days of simulation time using 32 regions (SLs), each with 10 locations (LPs) and each location with a population of 1000 (event messages). The LOC of 50\% and 90\% in DSB suggest that $50 \%$ and $90 \%$ of the trips the population performs are within the same region, respectively, which also suggests that the rest of the trips performed are across the regions (SL). Figure 5 shows the benchmark runtimes' three setups: (a) native without hypervisor, (b) DOM0 with 32 VCPUs and, (c) DOMU with 32 VCPUs along with DOM0 without computational 


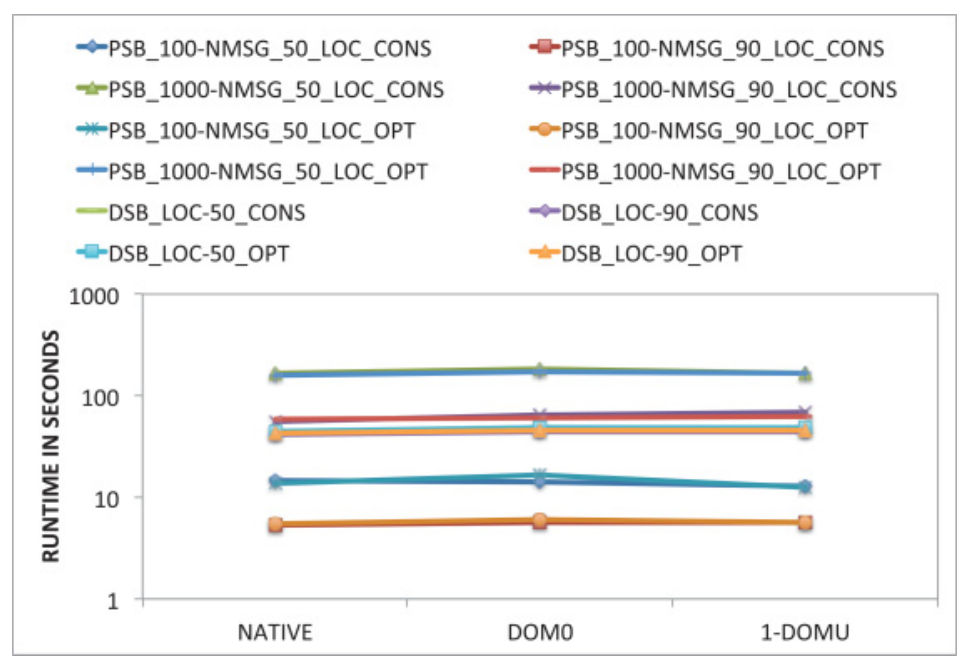

Fig. 5. Native, DOM0 and DOMU performance comparison on LTP.

load. The runtime results across all three setups were found to be almost identical for all benchmarks. This result is significant because it demonstrates that when the virtual resources exactly match the physical resources, the performance of native and VM platforms are almost identical, suggesting a very low overhead due to the presence of the hypervisor.

5.3.2. Configurability Options. With a hypervisor, the physical platform can host VMs of different capacities concurrently. This flexibility provides various options to the user for VM selection. For example: a physical platform with 32 CPU cores can host a single VM with 32 VCPUs or 2 VMs each with 16 CPU cores or 4 VMs each with 8 cores, and so on. The Cloud platforms utilize this feature, offering choices to the user with VMs of different capacities, and the VM usage cost is usually directly proportional to its compute capacity. Hence, it becomes necessary to empirically evaluate the performance of PDES applications across a range VM configurations. Figure 6 and Figure 7 presents the runtime performance trends of DSB with the increase in number of VMs on LTP and Amazon's EC2 Cloud platform, respectively. Note that even though the number of hosted VMs are different, the aggregate compute resource in each of our test VM configurations exactly matches the physical compute resource of the LTP. An interesting and common trend in both graphs is that the performance degrades with fewer VMs beyond 1, and the performance gets better with an increase in the number of VMs. This trend was also observed with PHOLD benchmarks. More information on this counterintuitive behavior can be found in Yoginath and Perumalla [2013a]. This trend affects the monetary cost in VM utilization, as the cost of a VM in the Cloud is predominantly determined by its compute capacity (number of VCPUs).

5.3.3. Cost vs. Performance on EC2. While the cost model for the utilization of Cloud compute resources makes economic sense, the options and related pricing of the offered VMs generally confound the user. This is more true if the user intends to use the Cloud resource for a parallel computing application, like PDES application. In this case, the monetary cost of executing the same PDES application among various configurable options of VM resources provides necessary insight. In Figure 8, we plot the cost of executing various PHOLD simulation scenarios on a cluster formed using various EC2 Cloud resources. In this graph, the left-most VM (on the X-axis) is the costliest, while 


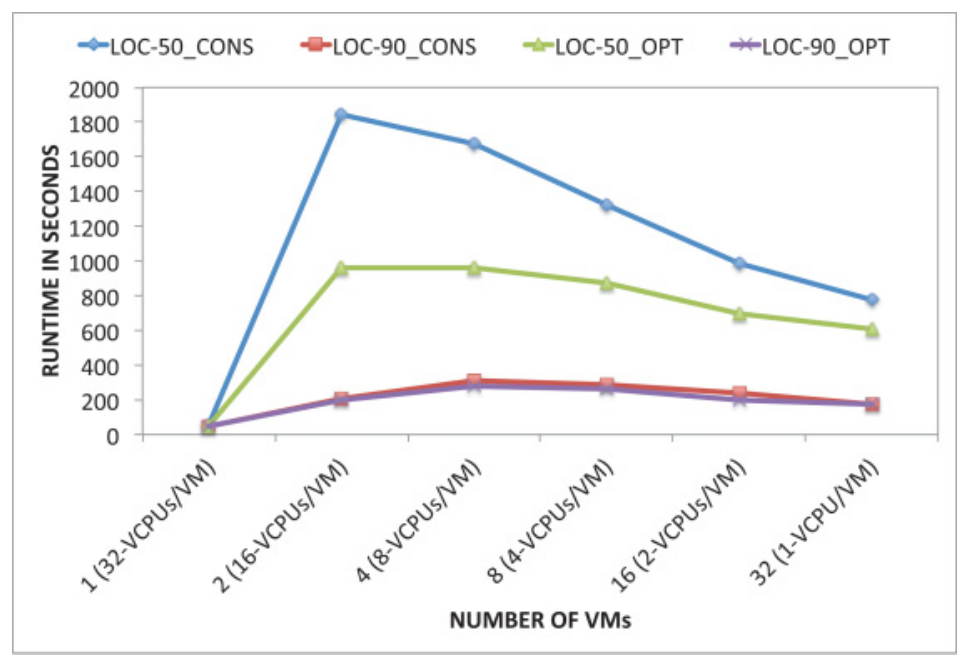

Fig. 6. Performance comparison with increase in the number of DOMs using DSB on LTP.



Fig. 7. Runtime performance of DSB on Amazon EC2 Cloud.

the right-most is the cheapest in terms of monetary cost. From this graph, we observe that the costliest resource might not yield the best performance, while the cluster of VMs formed by single-VCPU (m1.medium) seems to provide a better value for the cost. In Figure 9, we plot the runtime and the cost of one of the dense simulation PHOLD scenarios using conservative synchronization (with better runtime than its optimistic counterpart) for comparison. A similar trend was observed with the DSB benchmarks. We refer the interested reader to Yoginath and Perumalla [2013a] for more information.

5.3.4. Higher Multiplexing Ratios. Having learned that a cheaper cluster of single-VCPU VM would yield better performance than a costlier multi-VCPU VM, we further investigated the impact on PDES application performance with an increase in the compute resource multiplexing (virtual to physical) ratio. Note that, in all our previous performance discussions, we maintained a 1:1 multiplexing ratio, that is, the number 


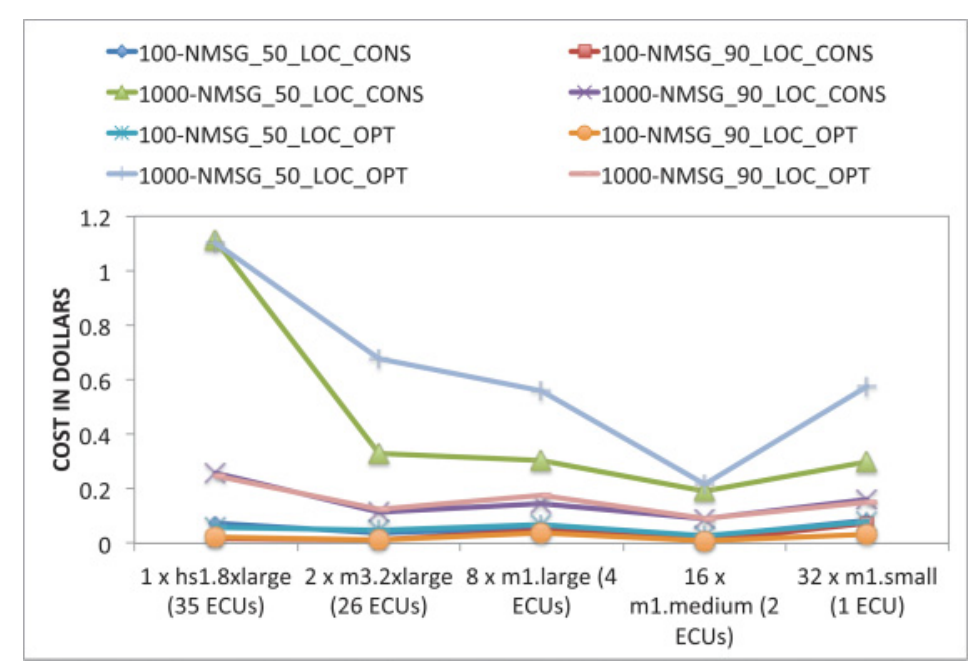

Fig. 8. Cost of running PHOLD benchmarks on Amazon EC2 Cloud.

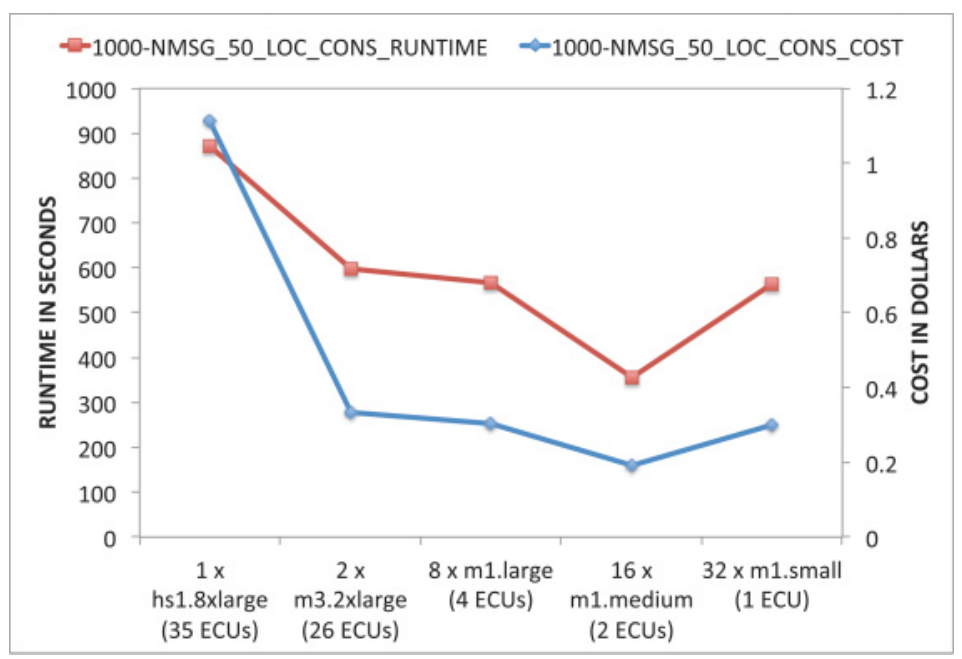

Fig. 9. Cost and runtime plots of a large-scale PHOLD benchmark scenario with $\mathrm{NLP}=100, \mathrm{NMSG}=1000$, $\mathrm{LOC}=50, \mathrm{LA}=1$ using a conservative synchronization scheme on Amazon EC2 Cloud.

of virtual resources hosted on a hypervisor were equivalent to number of physical resources utilized. This is an important study characteristic because the Cloud computing scales economically based on this concept. The understanding and a fact that not every VM hosted on a physical resource utilizes its compute resources continuously allows the vendors to host VMs whose aggregate compute resources surpass the actual number of physical compute resources. While, this oversubscription does not introduce any problems, when VMs are independent of each other or when the parallel tasks are embarassingly parallel, it makes a highly negative impact on a fine-grained PDES application's performance. This performance issue can be directly attributed to the virtual compute resource scheduling (hypervisor scheduling) strategy. Figure 10 captures this effect using PHOLD benchmarks over LTP, and these plots assert the need for a PDES-based hypervisor scheduler. 

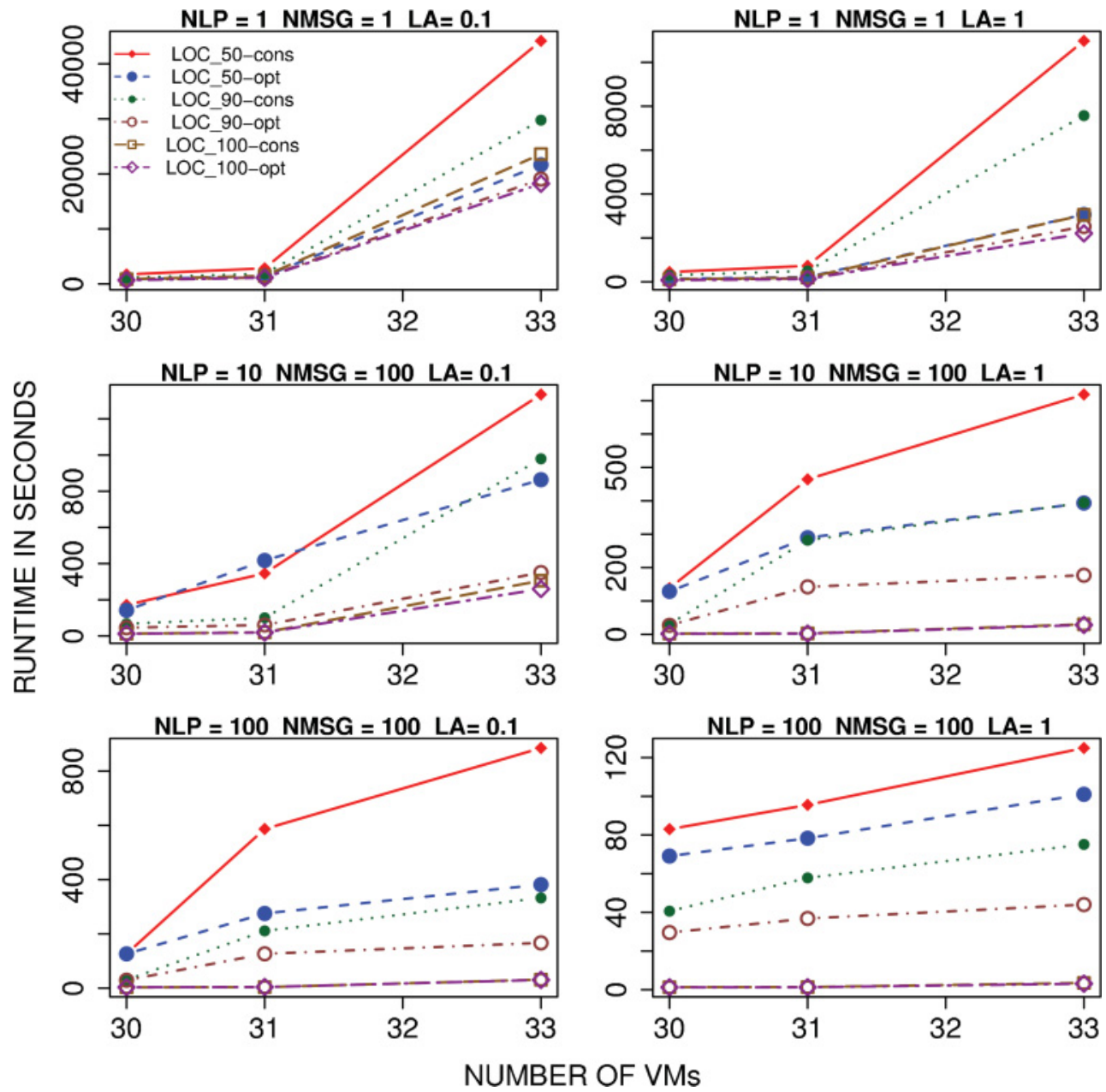

Fig. 10. CSX performance with increase in number of VMs.

In this set of experiments, the DOM0 was set up to use two VCPU cores, thus at any time instance 30 remnant PCPUs are available for a hosted VM or VMs. We launched single VCPU VMs equal to remnant PCPUs (30) and increased the number of VMs hosted until the number of VCPUs became $10 \%$ greater than the number of PCPUs. Figure 10 plots the runtimes for varying PDES loads for a mere $10 \%$ increase in the number of hosted VCPUs.

The top two graphs in Figure 10 plot performance runs with lowest possible computational load for varying communication loads and for varying lookaheads 0.1 (left) and 1.0 (right). These show the effect of VCPU scheduling in the absence of a significant computational load. These plots show several orders of magnitude of degradation in performance with a negligible increase in load. These readings constitute some of the worst possible performances that can be expected on a Cloud platform.

The bottom two graphs in Figure 10 plot performance runs with highest possible computational load for varying communication loads and for varying lookaheads of 0.1 (left) and 1.0 (right). This set of readings represents one of the best performances that 


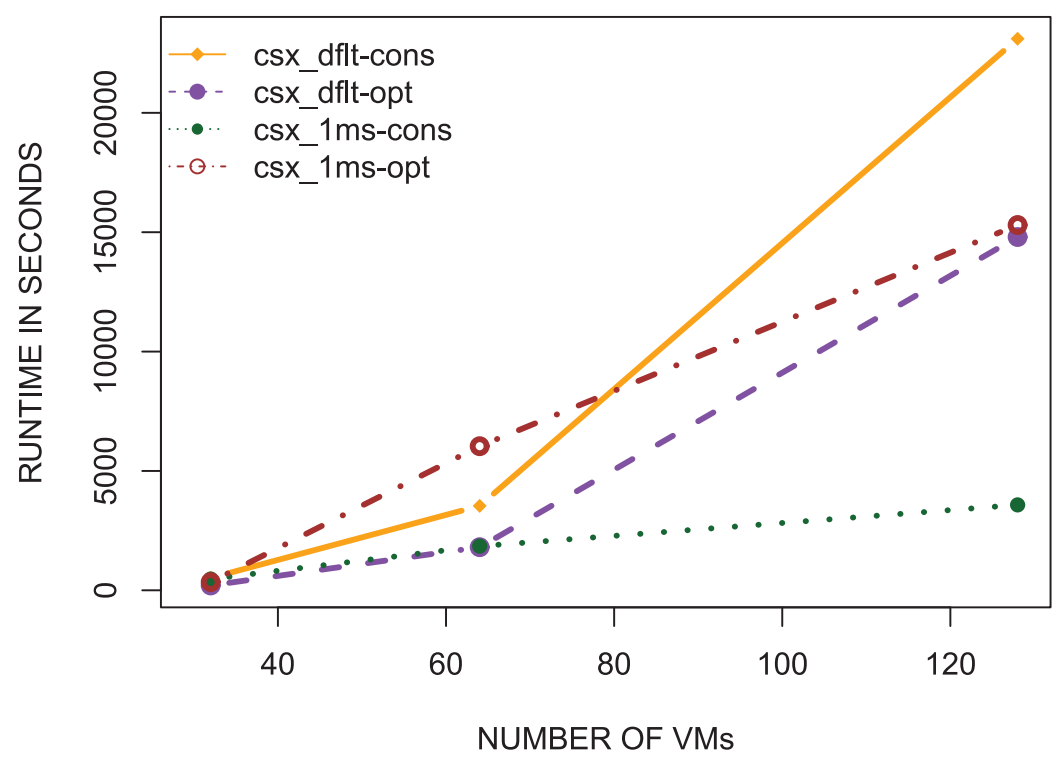

Fig. 11. Runtime performance of PHOLD for varying time slices.

PDES applications can expect on a Cloud platform. Yet, based on the communication load, the performance varies significantly. At worst, it is an order of magnitude slower, as shown by the plots for $\mathrm{LOC}=50 \%$ and 0.1 lookahead configuration.

The center two graphs in Figure 10 plot average computational load for varying communication loads and for varying lookaheads 0.1 (left) and 1.0 (right). This set of readings can be considered to represent an average behavior of most of the PDES applications. Performance degradation by several folds with an increase in VMs, especially with an increase in the communication load, highlights the high impact of scheduling on PDES application performance.

5.3.5. Lower Time Slice. We observed that the time slice provided to each VCPU during scheduling significantly alters the performance of the PDES application. Changing the time slices is made easy in the recent releases of Xen hypervisor using the $x l$ tool. By default, CSX provides a time slice of $30 \mathrm{~ms}$ in quantums of $10 \mathrm{~ms}$ tick size for each scheduled VCPU. The time slice can at most be reduced to $1 \mathrm{~ms}$ using the $x l$ tool. Figure 11 compares the runtimes of the PHOLD benchmark scenario (NLP=100, $\mathrm{NMSG}=100, \mathrm{LOC}=95, \mathrm{LA}=0.1$, and endtime $=1 \mathrm{e} 3$ ) for $\mathrm{CSX}$ with default time slice with CSX with 1ms time-slice. As seen in Figure 11, conservative synchronization performs extremely well with reduced time slice as evident in 128-VM and 64-VM scenarios. Close to an order-of-magnitude performance gain is in the 128-VM scenario. However, the same is not true while using the optimistic synchronization case. In the $128-\mathrm{VM}$ scenario using optimistic synchronization, a $1 \mathrm{~ms}$ time slice makes no difference in runtime when compared to a default time slice, and the performance suffers very badly in the 64-VM scneario. This is because of a high number of reversals (tens of millions) in the case of $1 \mathrm{~ms}$ time-slice runs compared to a lower (few hundred thousands) number of reversals, while using a default time slice. In the absence of high reversals, optimistic synchronization can be expected to perform better than conservative synchronization. Hence, all following performance runs use a CSX with a $1 \mathrm{~ms}$ time slice. 




Fig. 12. PSX and CSX comparison for PHOLD with $\mathrm{LA}=0.1$.

\subsection{PSX Performance Comparisons}

5.4.1. PSX and CSX Performance Comparison Using PHOLD Benchmarks. For this set of performance results, we use a PHOLD scenario with $\mathrm{NLP}=100, \mathrm{NMSG}=100$, and $\mathrm{LOC}=95$, and we host one $\mu$ sik SL on a VM. NLP $=100$ ensures that each VM/SL hosts $100 \mathrm{LPs}$. $\mathrm{NMSG}=100$ ensures that each LP exchanges 100 messages among its peers. Thus at any instance, a simulation scenario with $128 \mathrm{VMs}$ exchanges 1.28 million messages among $12,800 \mathrm{LPs}$. The locality (LOC) is set to $95 \%$, suggesting that $95 \%$ of the randomly generated messages are local, while the $5 \%$ are sent to a random peer LP hosted on other VM. Locality of $95 \%$ ensures that the SL has enough local events to process at any instance, hence the effect of scheduling could be minimal, as observed in Figure 10. For all PSX runs, the GVT_Threshold(GT) was kept at 10.

Figure 12 shows the plots of conservative and optimistic runs with lookahead of 0.1 . As the number of VMs hosted on the physical machine increases, both optimistic and conservative runtimes of the PSX perform well in comparison to their CSX counterparts. While the PSX using conservative synchronization suffers slightly, the PSX execution using optimistic synchronization performs very well with the increase in the number of VMs.

The plot on the left in Figure 12 is magnification of the initial set of points. They demonstrate the behavior of PSX with respect to CSX when the multiplexing ratio of VCPUs on to PCPUs are low. As seen, CSX performs best when no mismatch between PCPUs and VCPUs exist and suffers significantly even due to a slight mismatch. In contrast, the PSX suffers in the absence of the mismatch due to unnecessary overhead of writing LVTs to the hypervisor and performs better than CSX as the mismatch grows, as expected.

5.4.2. PSX and CSX Performance Comparison Using Disease Spread Benchmarks. This DSB benchmark scenario comprises $\mu$ sik SLs that correspond to region and LPs that correspond to locations. Each region ( $\mu$ sik SL) hosts multiple locations (LPs). Each region or SL is hosted on a VM. Here, the DSB scenario comprises 100 locations per region and a population of 100 per each location. Thus with $128 \mathrm{VMs}$, we simulate the disease 


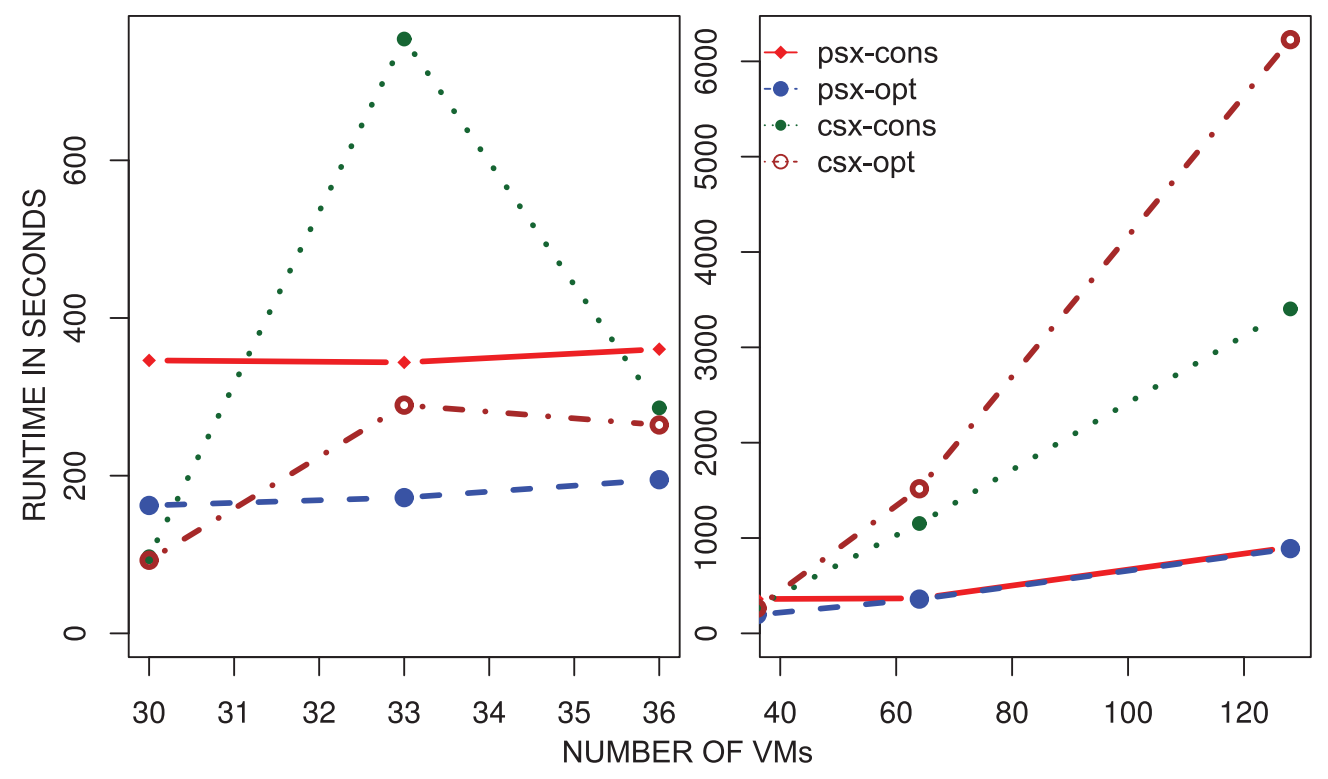

Fig. 13. PSX and CSX performance comparison for DSB with $\mathrm{LOC}=50$.

spread across a population of $1,280,000$ people across 128 regions, each with 100 localities. The simulation scenario studies the disease spread among the population over a week.

Figure 13 shows the runtime plots for $\mathrm{LOC}=50 \%$, suggesting that $50 \%$ of the population moves across regions. Both optimistic and conservative runs with PSX scheduler perform extremely well with the increase in the VMs. We also experimented with higher LOC percentages ( $\mathrm{LOC}=90 \%$ ), which limits the population movement across regions to $10 \%$ and observed similar performance trends. Similar to the PHOLD benchmark runs, the DSB benchmarks also show that when the physical and virtual compute resources match, the CSX performs slightly better than PSX.

5.4.3. PSX and CSX Performance Using SCATTER Benchmarks. As opposed to the two prior benchmarks that evaluated weak-scaling (increase in computational load with increase in number of $\mathrm{VMs}$ ), this benchmark evaluates strong-scaling behavior (computational load remains the same across all scenarios varying in terms of number of VMs used). The SCATTER benchmark simulates the vehicular traffic evacuation scenario of 3.2 million vehicles originating from 256 sources. Each vehicle makes its way across a $128 \times 128(16 \mathrm{~K})$ grid of intersections toward its destination (one of the 256 sinks), using Dijkstra's shortest-path algorithm. The vehicles were generated in source node at a rate of 50 vehicles/sink/hour for 1 hour. Vehicles injected by the sources placed on either side (left and right) move toward the sinks (top and bottom) across a road-network grid of $128 \times 128$ intersections. The same simulation scenario is executed on 32,64 , and $128 \mathrm{VMs}$. The intersections, sources, and sinks are modeled as LPs of PDES. The spatial decomposition ensured that equal numbers of intersection LPs, source LPs, and sink LPs were allotted to each $\mu$ sik SL hosted on a VM.

The corresponding performance plots are presented in Figure 14. The runtime of the optimistic plot for the PSX remains almost the same with the increase in number of VMs. The PSX conservative runtime also shows a similar trend, except when the number of VMs hosted is 128 , when its runtime slightly increases. In comparison, the 




Fig. 14. PSX and CSX performance comparison for the SCATTER vehicular traffic simulation scenario.

CSX runtime suffers as the number of VMs hosted increases. However, the CSX using optimistic synchronization is able to curtail the performance degradation significantly in comparison with its conservative synchronization.

5.4.4. PSX, CSX, and Native Performance Comparisons. In this section, we compare the performance benchmarks on the VM platform with the native Linux platform, on the same hardware device. For a fair comparison, the number of $\mu$ sik SL processes equivalent to number of VMs used (in VM setups) were spawned on Linux. The executions involving $128 \mathrm{VM}$ s using PHOLD, DSB, and SCATTER were used for comparison. The best runtime results, regardless of the PDES synchronization scheme exercised, was used for comparison.

As seen in Figure 15, the PHOLD benchmark runtime on Linux using 128 processes is several orders of magnitude faster than results from CSX and PSX. Though PSX is able to alleviate the performance degradation to a certain extent, it still is inefficient because the PHOLD benchmark is very fine grained and the low lookahead (0.1) requires frequent synchronization. This is in spite of optimistic synchronization trying its best to keep the runtime lower. The native runs are almost over an order of magnitude faster than a VM environment using PSX or CSX.

For the DSB benchmark, the best runtime with CSX is extremely bad; however, PSX has been able to significantly boost the performance of the DSB benchmark, bringing it closer to the native Linux performance. The DSB benchmark has a higher computational load in comparison with PHOLD. Even though the communication load $(\mathrm{LOC}=50)$ is higher, with efficient scheduling, both conservative and optimistic synchronizations perform very well.

For the SCATTER benchmark, PSX performs extremely well. While CSX is only a few times slower than native Linux runtime, PSX is very close to the native runtime performance. This is because the SCATTER scenario is computationally intensive, very well load balanced, and optimistic synchronization with zero-rollbacks yields very good performance. PSX with its LVT-based scheduling further improves the performance. 


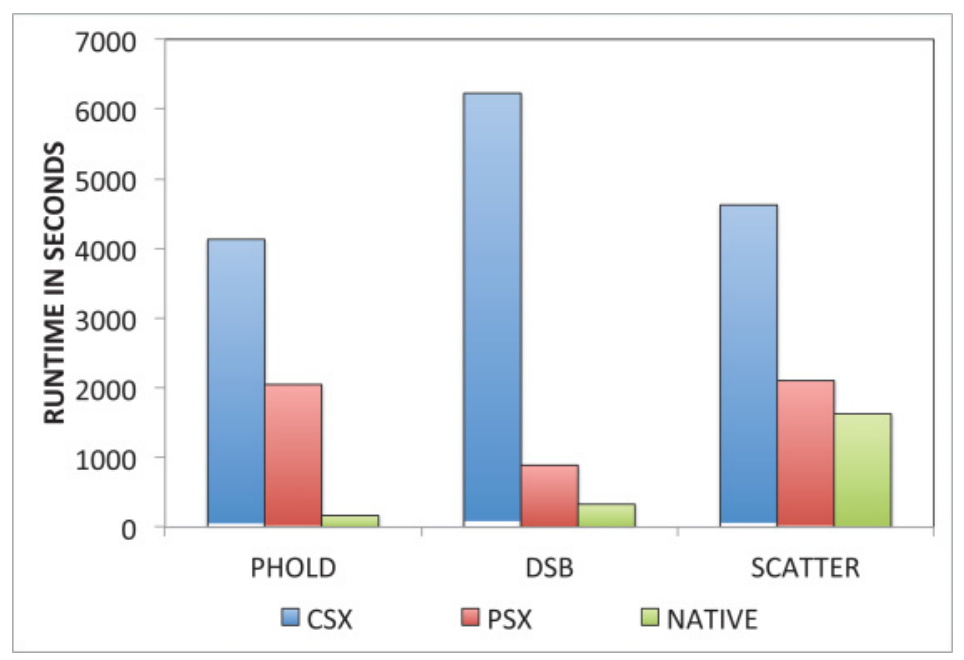

Fig. 15. PSX, CSX and Native Linux performance comparisons with PHOLD (LA=0.1), DSB $(L O C=50)$ and SCATTER benchmarks.

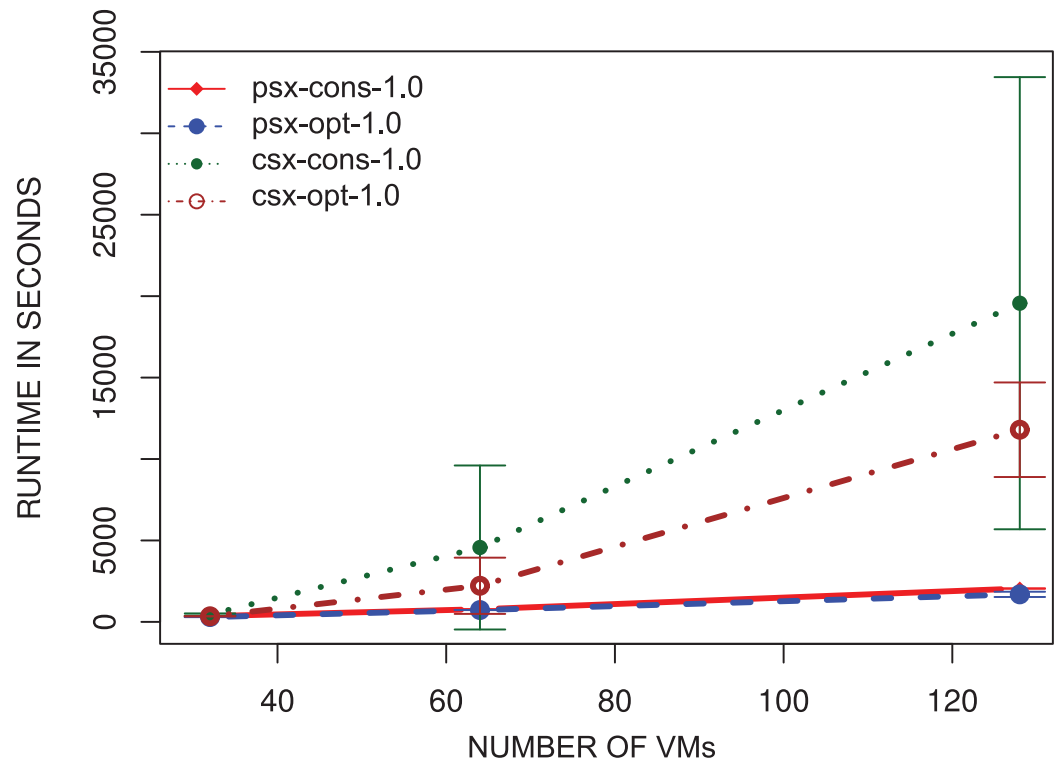

Fig. 16. PSX and CSX variance (with 95\% confidence interval) using PHOLD with 1 lookahead.

5.4.5. Variance in Performance. The data points of CSX showed high variance when the number of VCPUs multiplexed were greater than the number of PCPUs. Figure 16 plots the variance of data points of PSX and CSX runtimes in PHOLD benchmark, with lookahead 1. In our previous plots, we had used the best runtimes obtained using CSX plots from multiple runs for comparison with the runtimes of PSX. The observed behavior can be expected from CSX because of the VCPU scheduling strategy it uses. In contrast to CSX, the PSX readings show a very low variance regardless of the number of multiplexed VMs. 


\section{RELATED WORK}

Evaluation of high-performance computing applications on Cloud infrastructures has been reported in Jackson et al. [2010]; these applications are largely non-PDES, scientific codes such as Community Atmospheric Model (CAM). Network performance on Amazon EC2 data centers has been studied and an evaluation of the impact of virtualization on network parameters such as latency, throughput, and packet loss was discussed in Wang and Ng [2010], again in a non-PDES context. There is a good overview and discussion of generic utilization of Cloud infrastructure for PDES applications stressing the advantages and challenges it poses [D'Angelo 2011], which serves as a good motivation and background for PDES on Cloud platforms. The Master-Worker approach to distributed (and fault-tolerant) PDES [Park 2009] and optimistic Cloudbased execution [Malik et al. 2009, 2010; Fujimoto et al. 2010] are also related but complementary approaches, different from our support for the traditional PDES execution view, in which all processors are equal. We also adopt a unique approach by focusing at the lowest level, namely at the level of the hypervisor itself. Recently, Vanmechelen et al. [2012] reported evaluation of a set of conservative synchronization protocols on EC2, suggesting conservative algorithms that could perform better in the Cloud infrastructure. Overall, the area is nascent, and much additional research is needed to explore the space opened by the new metrics beyond raw speed of PDES execution.

The poor performance of certain high-performance computing applications has also been observed [Jackson et al. 2010]; customized solutions are being proposed that are not applicable to PDES, which fundamentally relies on virtual time order.

Incidentally, the Time-Warp Operating System [Jefferson et al. 1987] of the 1980s is one of the earliest works that addressed PDES performance issues by realizing the simulation scheduler (and related functionality) at the bottom-most hardware levels; however, this was limited to a single operating system as opposed to a hypervisor. There is also a superficial semblance to related work in VM-based network simulations [Yoginath and Perumalla 2011; Zheng and Nicol 2011; Yoginath et al. 2012]. However, VM-based network simulations are fundamentally different from PDES execution over VM platforms. In VM-based network simulations, the simulation time of each VM is determined by the hypervisor itself (in terms of computation time consumed by each VM, tracked and accounted by the hypervisor), whereas for PDES execution over VMs (discussed in this article), the virtual time used during VCPU scheduling corresponds to the user's simulation model.

While our implementation and experimentation have been performed in the context of the Xen [Chisnall 2007] hypervisor and the $\mu$ sik [Perumalla 2005] parallel/ distributed simulation kernel, the concepts developed in this article for VM-based PDES are sufficiently general, and can be applied to other hypervisors and parallel discrete event simulators.

\section{SUMMARY}

We started by listing the advantages of using Cloud platforms for PDES applications. We found that the runtime performance (one of the core reasons for PDES execution) of a PDES application suffered acutely on the Cloud platforms. To understand and unravel the performance issues of PDES application payloads on Cloud platforms, we undertook an extensive PDES performance study on a custom-built hardware with a hypervisor capable of hosting hundreds of VMs. Several performance behaviors, such as (a) almost similar runtime performance of PDES application over VM and native platform, when virtual and physical resources match; (b) a counterintuitive performance trend while using various compositions of VM compute resources for PDES execution; (c) monetary 
implications of observed performance; (d) severe performance degradation with a slight increase $(10 \%)$ in the virtual to physical multiplexing ratio; and (e) better performance of PDES using smaller time slices in VCPU scheduling, were discovered.

As a generic guideline when executing a PDES application for the Cloud, if the number of processor cores of the physical system on which VMs are hosted is known, then use a single VM with the number of VCPUs equal to the number of processor cores. In the absence of these details, the best performance for the monetary cost involved can be obtained by using a cluster of VMs, each with a single VCPU. Note that this guideline is valid when the virtual compute resources exactly match the physical compute resources. Even a slight increase (less than 10\%) in the virtual to physical multiplexing ratio yields poor performance. We discovered that the poor performance of the PDES application was related to the hypervisor scheduling policy.

After recognizing scheduling policy mismatch in Cloud platforms as the reason for poor PDES performance, we designed, implemented, and extensively evaluated the performance of a new PDES specific scheduler. We used different scenario configurations of synthetic PHOLD, disease spread simulation benchmarks, and vehicular traffic simulation benchmarks to evaluate the performance. We demonstrated a significant speedup using a PSX scheduler over a CSX scheduler across all the benchmark runs. Note that all the performance comparisons were done with CSX using small time slices; hence, the speedup against CSX with default time-slice configurations can be expected to be much higher (order of magnitude). We also compared the runtime performance of our three simulation benchmarks involving $128 \mathrm{VMs}$ with 128 processes hosted on a Linux machine with the same hardware, and demonstrated that a tremendous reduction in performance degradation can be achieved in VM-based execution platforms. We also demonstrated the high variance of PDES application runtime with CSX, and very low variance with PSX execution setups.

\section{CONCLUSION AND FUTURE WORK}

With the proliferation of Cloud and VM-based platforms for parallel computing, it is now possible to execute PDESs over multiple VMs, in contrast to executing in native mode directly over hardware, as has been traditionally done over the past decades. However, while most VM-based platforms are optimized for general workloads, PDES execution exhibits unique dynamics significantly different from other workloads. Here, we present results that identify the gross deterioration of the runtime performance of VM-based PDES simulations when executed using traditional VM schedulers, quantitatively showing the bad scaling properties of the scheduler as the number of VMs is increased. The mismatch is fundamental in nature in the sense that any fairness-based VM scheduler implementation would exhibit this mismatch with PDES runs.

To solve this mismatch, a new algorithm has been presented for PDES-specific scheduling of VMs by a hypervisor. The algorithm schedules VMs primarily by their LVT order, and incorporates mechanisms that prevent deadlocks that are otherwise possible in a purely LVT-based scheduling. The new scheduler has been implemented and exercised in an actual hypervisor system (Xen) that is popularly used in major Cloud platforms worldwide.

Experimental results have been documented from detailed experiments with multiple discrete event models over a range of scenarios (with different lookahead values, interprocessor event exchange frequencies, and conservative and optimistic synchronization), all of which show (a) the high variability and suboptimality of the default credit-based VM scheduler that is PDES-agnostic, and (b) the well-behaved scalability and significantly faster execution of our new algorithm. 
The study and results presented here are among the first to evaluate the characteristics of Cloud and VM-based PDES in detail, and the first to propose a deadlock-free PDES-customized hypervisor scheduler.

The results are timely due to the great appeal of commercial Cloud offerings that many find to be very user friendly and convenient to access and manage. Future work of interest includes incorporating and benchmarking the support for dynamic growth and shrinkage of physical processors allocated to a PDES run dynamically during its execution. Using CPU-Pools support of Xen to get our PDES-specific scheduler into the realms of current Cloud-computing infrastructure can make it accessible by more PDES application developers and users. PDES runs may also benefit from the inclusion of a network metric in the specification of the abstract computational unit for VMs, the absence of which leaves the computation highly sensitive to the vagaries of virtual network devices. Cloud-specific synchronization algorithms may also be needed to be resilient to variations in virtual network latencies.

\section{REFERENCES}

David Chisnall. 2007. The Definitive Guide to the Xen Hypervisor. Pearson Education, Inc., Prentice-Hall, Upper Saddle, NJ.

Edward G. Coffman, Melanie Elphick, and Arie Shoshani. 1971. System deadlocks. ACM Computing Surveys (CSUR) 3, 2, 67-78.

G. D'Angelo. 2011. Parallel and distributed simulation from many cores to the public cloud. In Proceedings of the 2011 International Conference on High Performance Computing and Simulation (HPCS). 14-23. DOI : http://dx.doi.org/10.1109/HPCSim.2011.5999802

R. M. Fujimoto. 1990. Performance of time warp under synthetic workloads. In Proceedings of $22 \mathrm{nd}$ SCS Multiconference on Distributed Simulation.

Richard M. Fujimoto, Asad Waqar Malik, and A. Park. 2010. Parallel and distributed simulation in the cloud. SCS M\&S Magazine 3, 1-10.

K. R. Jackson, L. Ramakrishnan, K. Muriki, S. Canon, S. Cholia, J. Shalf, Harvey J. Wasserman, and N. J. Wright. 2010. Performance analysis of high performance computing applications on the Amazon web services cloud. In Proceedings of the 2010 IEEE 2nd International Conference on Cloud Computing Technology and Science (CloudCom). 159-168. DOI : http://dx.doi.org/10.1109/CloudCom.2010.69

D. Jefferson, B. Beckman, F. Wieland, L. Blume, and M. Diloreto. 1987. Time warp operating system. SIGOPS Operating Systems Review 21, 5, 77-93. DOI : http://dx.doi.org/10.1145/37499.37508

A. W. Malik, A. Park, and R. M. Fujimoto. 2009. Optimistic synchronization of parallel simulations in cloud computing environments. In Proceedings of the IEEE International Conference on Cloud Computing. 49-56. DOI : http://dx.doi.org/10.1109/CLOUD.2009.79

A. W. Malik, A. Park, and R. M. Fujimoto. 2010. An optimistic parallel simulation protocol for cloud computing environments. SCS M\&S Magazine 4, 1-9.

Jeanna N. Matthews, Eli M. Dow, Todd Deshane, Wenjin Hu, Jeremy Bongio, Patrick F. Wilbur, and Brendan Johnson. 2008. Running Xen: A Hands-On Guide to the Art of Virtualization. Prentice-Hall, Upper Saddle, NJ.

Peter Mell and Timothy Grance. 2011. The NIST definition of cloud computing (draft). NIST Special Publication $800,145,7$.

Alfred J. Park. 2009. Master/Worker Parallel Discrete Event Simulation. Ph.D. Dissertation. Georgia Institute of Technology, Atlanta, GA.

Kalyan S. Perumalla. 2005. $\mu$ sik—a micro-kernel for parallel/distributed simulation systems. In Proceedings of the Workshop on Principles of Advanced and Distributed Simulation (PADS'05). IEEE, 59-68.

Kalyan S. Perumalla and Sudip K. Seal. 2012. Discrete event modeling and massively parallel execution of epidemic outbreak phenomena. Simulation 88, 7, 768-783.

Kurt Vanmechelen, Silas De Munck, and Jan Broeckhove. 2012. Conservative distributed discrete event simulation on Amazon EC2. In Proceedings of the IEEE/ACM International Symposium on Cluster, Cloud and Grid Computing (CCGRID'12). IEEE Computer Society, Washington, DC, 853-860. DOI : http://dx.doi.org/10.1109/CCGrid.2012.73

Guohui Wang and T. S. Eugene Ng. 2010. The impact of virtualization on network performance of Amazon EC2 data center. In Proceedings of the 29th Conference on Information Communications (INFOCOM'10). IEEE Press, Piscataway, NJ, 1163-1171. http://dl.acm.org/citation.cfm?id=1833515.1833691 
Srikanth B. Yoginath and Kalyan S. Perumalla. 2008. Parallel vehicular traffic simulation using reverse computation-based optimistic execution. In Proceedings of the 22nd Workshop on Principles of Advanced and Distributed Simulation (PADS'08). IEEE, 33-42.

Srikanth B. Yoginath and Kalyan S. Perumalla. 2009. Reversible discrete event formulation and optimistic parallel execution of vehicular traffic models. International Journal of Simulation and Process Modelling 5, 2 (2009), 104-119.

Srikanth B. Yoginath and Kalyan S. Perumalla. 2011. Efficiently scheduling multi-core guest virtual machines on multi-core hosts in network simulation. In Proceedings of the 2011 IEEE Workshop on Principles of Advanced and Distributed Simulation (PADS'11). IEEE, 1-9.

Srikanth B. Yoginath and Kalyan S. Perumalla. 2013a. Empirical evaluation of conservative and optimistic discrete event execution on cloud and VM platforms. In Proceedings of the 2013 ACM SIGSIM Conference on Principles of Advanced Discrete Simulation (SIGSIM-PADS'13). ACM, New York, NY, 201-210. DOI : http://dx.doi.org/10.1145/2486092.2486118

Srikanth B. Yoginath and Kalyan S. Perumalla. 2013b. Optimized hypervisor scheduler for parallel discrete event simulations on virtual machine platforms. In Proceedings of the 6th International ICST Conference on Simulation Tools and Techniques (SimuTools'13). Institute for Computer Sciences, Social-Informatics and Telecommunications Engineering, Brussels, Belgium, 1-9. http://dl.acm.org/citation.cfm?id=2512734.2512735

Srikanth B. Yoginath, Kalyan S. Perumalla, and Brian J. Henz. 2012. Taming wild horses: The need for virtual time-based scheduling of VMs in network simulations. In Proceedings of the 2012 IEEE 20 th International Symposium on Modeling, Analysis \& Simulation of Computer and Telecommunication Systems (MASCOTS'12). IEEE, 68-77.

Yuhao Zheng and David M. Nicol. 2011. A virtual time system for openvz-based network emulations. In Proceedings of the 2011 IEEE Workshop on Principles of Advanced and Distributed Simulation (PADS'11). IEEE, 1-10.

Received January 2014; revised March 2015; accepted March 2015 